\title{
Wide-Field Retinotopy Defines Human Cortical Visual Area V6
}

\author{
Sabrina Pitzalis, ${ }^{1,2}$ Claudio Galletti, ${ }^{3}$ Ruey-Song Huang, ${ }^{4}$ Fabiana Patria, ${ }^{1}$ Giorgia Committeri, ${ }^{1,5}$ Gaspare Galati, ${ }^{1,2,5}$ \\ Patrizia Fattori, ${ }^{3}$ and Martin I. Sereno ${ }^{4}$ \\ ${ }^{1}$ NeuroImaging Laboratory and 2 Laboratory of Neuropsychology, Santa Lucia Foundation, Istituto di Ricovero e Cura a Carattere Scientifico, 00179 Rome, \\ Italy, ${ }^{3}$ Department of Human and General Physiology, University of Bologna, I-40127 Bologna, Italy, ${ }^{4}$ Cognitive Science 0515, University of California, San \\ Diego, La Jolla, California 92093-0515, and 5 Department of Clinical Sciences and Bioimaging, University Gabriele d’Annunzio, 66013 Chieti, Italy
}

The retinotopic organization of a newly identified visual area near the midline in the dorsalmost part of the human parieto-occipital sulcus was mapped using high-field functional magnetic resonance imaging, cortical surface-based analysis, and wide-field retinotopic stimulation. This area was found in all 34 subjects that were mapped. It represents the contralateral visual hemifield in both hemispheres of all subjects, with upper fields located anterior and medial to areas V2/V3, and lower fields medial and slightly anterior to areas V3/V3A. It contains a representation of the center of gaze distinct from $\mathrm{V} 3 \mathrm{~A}$, a large representation of the visual periphery, and a mirror-image representation of the visual field. Based on similarity in position, visuotopic organization, and relationship with the neighboring extrastriate visual areas, we suggest it might be the human homolog of macaque area V6, and perhaps of area M (medial) or DM (dorsomedial) of New World primates.

Key words: parieto-occipital cortex; extrastriate areas; dorsal visual stream; visual topography; cortical flattening; brain mapping

\section{Introduction}

Several dozen visual areas have been described so far in nonhuman primates (Felleman and Van Essen, 1991; Kaas and Krubitzer, 1991; Sereno and Allman, 1991; Lewis and Van Essen, 2000). Thanks to neuroimaging methods, several of these areas have been mapped in humans (Watson et al., 1993; Sereno et al., 1995, 2001; Tootell et al., 1995, 1997; DeYoe et al., 1996; McKeefry and Zeki, 1997; Tootell and Hadjikhani, 2001). In particular, areas V1, V2, V3, VP, V3A, V4v, and middle temporal (MT)/V5 have been named in humans based on homologies with nonhuman primate areas (for review, see Sereno, 1998; Sereno and Tootell, 2005). One prominent omission in that list is a human homolog of macaque area V6.

Area V6 has been described in macaque monkeys (see Fig. 1) on functional and connectional criteria (Galletti et al., 1996, 1999a, 2001, 2005). Macaque V6 is located in the depths of the parieto-occipital sulcus (POS) and contains a retinotopic map of the entire contralateral hemifield. In contrast to many extrastriate areas, V6 lacks an emphasis on the central visual field (Colby et al., 1988), a feature V6 shares with owl monkey area M (medial) (Allman and Kaas, 1976). V6 overlaps part of a cortical region in macaques originally named parieto-occipital

Received Aug. 4, 2005; revised May 1, 2006; accepted May 1, 2006

This work was supported by grants from Ministero dell'Istruzione, dell'Università e della Ricerca, and Fondazione del Monte di Bologna e Ravenna, Italy, and by National Science Foundation Grant NSF BCS 0224321. We thank Gisela Hagberg at the Fondazione Santa Lucia Istituto di Ricovero e Cura a Carattere Scientifico for help with the pulse sequence; R. Buxton, L. R. Frank, T. T. Liu, L. May, and E. C. Wong at the University of California, San Diego Functional MRI Center for development and support of image reconstruction software, pulse sequences, and scanner hardware; and Don Hagler for help with analysis software.

Correspondence should be addressed to Martin I. Sereno, Cognitive Science 0515, University of California, San Diego, La Jolla, CA 92093-0515. E-mail: sereno@cogsci.ucsd.edu.

DOI:10.1523/JNEUROSCI.0178-06.2006

Copyright $\odot 2006$ Society for Neuroscience $\quad$ 0270-6474/06/267962-12\$15.00/0
(PO) (Gattass et al., 1986; Colby et al., 1988) (see Discussion). In contrast to macaque V6 and owl monkey $\mathrm{M}, \mathrm{PO}$ as originally described lacked a representation of the central $20^{\circ}$ and contained a complex hemifield representation broken into several discontinuous parts (Gattass et al., 1986). Dorsal and anterior to macaque V6, partially overlapping the anterior part of PO, is another visual area, V6A (see Fig. 1) (Galletti et al., 1996). In contrast to V6, V6A is not retinotopically organized (Galletti et al., 1999b, 2005).

In humans, a "V6-complex," located in the parieto-occipital region, has been tentatively defined on functional criteria. By analogy with the monkey data, more ventral activity has been labeled V6, and more dorsal activity V6A (De Jong et al., 2001; Simon et al., 2002; Dechent and Frahm, 2003). However, in contrast with macaque V6 (and owl monkey area M), the putative human V6 showed a complete (Portin and Hari, 1999) or partial (Dechent and Frahm, 2003) lack of retinotopy in magnetoencephalography (MEG) and functional magnetic resonance imaging (fMRI) studies, respectively. That contrast with the data from nonhuman primates, however, may have been attributable to technical reasons, because nearby simultaneously active sources generated by multiple visual areas are difficult to disentangle using MEG, and both studies tested only the central $\left(<15^{\circ}\right)$ part of the visual field. Because monkey V6 deemphasizes the center of the visual field (Galletti et al., 1999a), here we used wide field retinotopic stimuli, reaching up to $55^{\circ}$ of eccentricity. These experiments uncovered the existence of an area in the dorsal parieto-occipital sulcus with retinotopic organization and position with respect to other extrastriate areas similar to those of macaque area V6.

Preliminary data have been published previously in abstract form (Pitzalis et al., 2004a,b). 


\section{Materials and Methods}

\section{Visual stimuli and experimental set-up}

Phase-encoded retinotopic stimuli were similar to those used previously to map the visuotopic organization in cortical visual areas (Sereno et al., 1995; Tootell et al., 1997). They consisted of high-contrast light- and dark-colored checks flickering in counterphase in either a ray- or a ringshaped configuration (polar angle and eccentricity, respectively). Each subject was presented with periodic stimuli (64 s/cycle, eight cycles/ scan), varying in stimulus eccentricity or polar angle, in at least two pairs of scans. Stimuli moved slowly and continuously, and checks reversed between light and dark at a rate of $8 \mathrm{~Hz}$. Subjects viewed polar angle stimuli moving in both clockwise (CW) and counterclockwise (CCW) directions. We adjusted the stimuli slightly to respect the distinctive characteristics of macaque V6 (and owl monkey area M). First, we used thin retinotopic stimuli to avoid saturation of the phase-encoded signal, because receptive fields in a putative human V6 would be expected to be larger than those in lower tier cortical areas (Galletti et al., 1999a). Second, given the shallower slope in the cortical magnification factor in macaque area V6 (Galletti et al., 1999a), eccentricity mapping stimuli were moved at a uniform velocity $(\sim 1 \%$, instead of increasing velocity as eccentricity increased) in an attempt to stimulate equal amounts of human V6 at different eccentricities, and to more clearly reveal the organization of the periphery in areas that emphasize it (check size remained logarithmically scaled with eccentricity). Finally, in some of the 3T scans, the flickering checkerboards in the ring- and ray-shaped apertures were replaced with naturalistic videos (e.g., episodes of the television action program Xena: Warrior Princess) presented inside rotating wedge-shaped masks and contracting or expanding ring-shaped masks. Subjects were required to fixate on the center cross while attending to the movies. Compared with checkerboards, the videos attract more attention, have spatiotemporal statistics closer to real world stimulation, and have been found to strongly activate both lower and higher visual areas in humans (Sereno et al., 2004).

We also greatly increased the size of the visual field that was stimulated (from a minimum of 102 up to $110^{\circ}$ in total visual extent). Stimuli were projected into the magnet onto a back-projection screen. The screen was viewed directly, not via a mirror. It was placed very close to the subject at a distance of 10-12 cm and was slightly adjusted by each subject so that they could comfortably fixate and focus on a central point on the screen without blurring [cf. Cheng et al. (1995), who used a smaller display and lenses to achieve a similar result]. At this short distance, polar angle and eccentricity visual stimuli subtended up to $100( \pm 50)^{\circ}$ horizontally, 80 $( \pm 40)^{\circ}$ vertically, and $110( \pm 55)^{\circ}$ in an oblique direction in the visual field. Brain-mapping studies on the human visual system have typically used much smaller visual stimuli $\left(8-12^{\circ}\right.$ eccentricity from the fixation point); consequently, these stimuli do not directly activate much of the periphery in many cortical visual areas (Sereno et al., 1995, 2001; Tootell et al., 1997, 1998).

The very large stimuli used here also help to deal with a possible confound in fMRI mapping studies caused by surround inhibition. fMRI studies in nonhuman primate studies suggest that when a local part of the visual cortex is stimulated, surrounding parts of a cortical visual map that have not been directly retinally stimulated show both a reduction in single unit activity as well as a reduced blood oxygen level-dependent (BOLD) fMRI signal (Brewer et al., 2002). In phase-encoded retinotopic mapping studies, cortical map regions that are never directly activated by the retina, but which are near periodically activated regions (e.g., retinotopic cortical map representations of visual space just beyond the peripheral edge of a rotating wedge) will therefore also generate a periodic signal (Sereno and Tootell, 2005). However, this signal will have a phase offset of $180^{\circ}$ from the veridical phase of the periodic signal in the nearby retinally stimulated region because the BOLD signal in these regions would be reduced every time the stimulus sweeps by. By stimulating most of the visual field, this misleading signal, that is, misleading for the purposes of retinotopic mapping, is greatly reduced.

An additional three scans were acquired to localize the motionsensitive area MT/V5 (MT mapping). We used the same stimulus used previously to map the human area MT (Tootell et al., 1995). In a block design sequence (on-off), moving $(7 \%$ s) and stationary patterns (con- centric white rings surrounding the central fixation point on a light-gray background, 0.2 cycles $/{ }^{\circ}$, duty cycle $=0.2$ ) were alternated in $32 \mathrm{sepochs}$ for 8 cycles/scan. During the on phase, the rings moved either inward (contracting) or outward (expanding). To increase functional specificity, the stimulus luminance contrast was low, as in the study by Tootell et al., (1995).

All experiments used passive viewing and subjects were required to maintain fixation throughout the period of scan acquisition. Head motion was minimized in most cases by using a bite bar with an individually molded dental impression mounted on a 6-degrees-of-freedom locking Plexiglas arm (Sereno et al., 2001). Subjects were instructed not to forcibly bite the impression but rather to use it as a reference. Subjects' heads were also stabilized with foam pads, and allowed to settle for a few minutes before the bite bar arm was fixed in position. Interior surfaces were covered with black velvet to eliminate reflections. Visual stimuli were projected using an XGA (extended graphics array) video projector $(1024 \times 768,72 \mathrm{~Hz}, 10-15$ pixels per degree of visual angle $)$ whose standard lens had been replaced with a 7.38-12.3 foot focal length XtraBright zoom lens (Buhl Optical, Pittsburgh, PA) to achieve small highresolution images on a screen inside the bore (a distance of 3-4 m).

\section{Imaging parameters}

All participants $(n=34)$ with (or corrected to) emmetropia gave their informed written consent before the scanning session, and all procedures were approved by the local Ethics and Human Subjects Committees. The MR examinations were conducted at the Santa Lucia Foundation (Rome, Italy) on a 1.5T Siemens Vision MR scanner (Siemens Medical Systems, Erlangen, Germany) and at the University of California, San Diego (San Diego, CA) fMRI Center on a Varian (Palo Alto, CA) 3T scanner (INOVA, San Diego, CA) and a GE 3T scanner (Signa EXCITE short bore; GE Medical Systems, Milwaukee, WI), all equipped for echo-planar imaging (EPI). Single shot EPI images were collected using a Small Flex quadrature surface radiofrequency coil placed over occipital and parietal areas (Siemens), a small transmit-receive end-capped birdcage head coil designed and fabricated by Eric Wong (Varian), and an eight-channel receive-only head coil (GE Medical Systems). MR slices were 3.5-4 mm thick, with an in-plane resolution ranging from $3.0 \times 3.0$ to $3.75 \times 3.75$ $\mathrm{mm}$, oriented approximately parallel to the calcarine fissure. This voxel size strikes a compromise between sufficient signal-to-noise and the ability to assign activations to the proper sides of the sulci and gyri. Each scan took either $256 \mathrm{~s}$ (two-condition experiments for MT+ mapping) or $512 \mathrm{~s}$ (retinotopy), with a repetition time (TR) $=2000$ or $4000 \mathrm{~ms}$. Each scan included 128 or 256 single-shot EPI images per slice in 16-32 contiguous slices [1.5T: echo time $(\mathrm{TE})=42$, flip angle $=90,64 \times 64$ matrix, bandwidth $=926 \mathrm{~Hz} /$ pixel; $3 \mathrm{~T}: \mathrm{TE}=26$, flip angle $=90$, bandwidth $=$ $1953 \mathrm{~Hz} /$ pixel, $64 \times 64$ matrix]. In each scan, the first $8 \mathrm{~s}$ of the acquisition was discarded from data analysis to achieve a steady state. A total of 423 functional scans were performed on the 34 subjects ( 319 scans to map the retinotopic visual areas, and 104 scans to map MT/V5).

The cortical surface of each subject was reconstructed from a pair of structural scans [T1-weighted magnetization-prepared rapidacquisition gradient echo (MPRAGE); TR $=11.4 \mathrm{~ms}$; TE $=4.4 \mathrm{~ms}$; flip angle $=108 ; 1 \times 1 \times 1 \mathrm{~mm}$ resolution] taken in a separate session using a head coil. The last scan of each functional session was an alignment scan (also MPRAGE, $1 \times 1 \times 1 \mathrm{~mm}$ or $1 \times 1 \times 2 \mathrm{~mm}$ ) acquired with the surface coil or head coil in the plane of the functional scans. The alignment scan was used to establish an initial registration of the functional data with the surface. Additional affine transformations that included a small amount of shear were then applied to the functional scans for each subject using blink comparison with the structural images to achieve an exact overlay of the functional data onto each cortical surface.

\section{Data analysis}

Processing of functional and anatomical images of the human data were performed using FreeSurfer (Dale et al., 1999; Fischl et al., 1999a) (http:// surfer.nmr.mgh.harvard.edu/; http://kamares.ucsd.edu/ sereno/csurf/ tarballs).

Anatomical image processing. The two high-resolution structural images obtained from each subject were manually registered using blink 
comparison, and then averaged, to enhance signal-to-noise ratio. The skull was stripped off by shrinking a stiff deformable template onto the brain images, the gray/white matter boundary was estimated with a region-growing method, and the result was tessellated to generate a surface that was refined against the MRI data with a deformable template algorithm. By choosing a surface near the gray/white matter border (rather than near the pial surface, where the macrovascular artifact is maximal), we were able to assign activations more accurately to the correct bank of a sulcus. The surface was then unfolded by reducing curvature while minimizing distortion in all other local metric properties. We completely flattened the inflated occipital lobe after first cutting it off posterior to the Sylvian fissure, and making an additional cut along the calcarine fissure.

Stereotaxic coordinates were calculated through an automatic nonlinear stereotaxic normalization procedure (Friston et al., 1995), performed using the statistical parametric mapping (SPM99) software platform (Wellcome Department of Cognitive Neurology, London, UK), implemented in MATLAB (MathWorks, Natick, MA). The template image was based on average data provided by the Montreal Neurological Institute (MNI) (Mazziotta et al., 1995). Talairach coordinates (Talairach and Tournoux, 1988) were also calculated through an automated nonlinear transformation (for a discussion on functional localization in the human brain, see Brett et al., 2002, and for more details on the applied MNI to Talairach transformation, see http://www.mrc-cbu.cam.ac.uk/Imaging/ Common/mnispace.shtml).

Functional image processing. Analysis methods were similar to previous studies (Sereno et al., 1995; Tootell et al., 1997; Hagler and Sereno, 2006). Raw data were first screened for motion artifacts; five scans were rejected from additional analysis on this basis. Motion correction was performed using the AFNI (Analysis of Functional NeuroImages) 3dvolreg (3T data). Data from phase-encoded retinotopic mapping experiments as well as two-condition experiments were analyzed by Fourier transforming the MR time course from each voxel (after removing constant and linear terms). This generates a vector with real and imaginary components for each frequency that defines an amplitude and phase of the periodic signal at that frequency. To estimate the significance of correlation of BOLD signal with the stimulus frequency (eight cycles per scan), the squared amplitude of the signal at the stimulus frequency is divided by the mean of squared amplitudes at all other "noise" frequencies (excluding low-frequency signals caused by residual head motion and harmonics of the stimulus frequency). This ratio of two $\chi^{2}$ statistics follows the F-distribution (Larsen and Marx, 1986), and with degrees of freedom equal to the number of time points, can be used to calculate a statistical significance $p$ value. Harmonics were excluded from the analysis because a wave of surround inhibition preceding and following the cortical representation of the moving stimulus can generate a second harmonic in retinally stimulated cortex (this is a different effect than the beyond-thestimulus-edge phase inversion described above (Sereno and Tootell, 2005).

The phase of the signal at the stimulus frequency was used to map retinotopic coordinates (polar angle or eccentricity). In standard blockdesign analysis, pseudocolor scales are usually used to represent the amplitude of the response (after masking the data with a significance threshold). In mapping studies, pseudocolor is also used to represent the phase of the response. To concentrate the viewer's attention on the phase, we modulate the saturation of the color as a function of the signal amplitude using a sigmoid function. The sigmoid function was arranged so that visibly saturated phase colors begin to emerge from the gray background at a threshold of $p<10^{-2}$. The data at most activated cortical surface points has much higher significance values $\left(p<10^{-5}\right.$ to $\left.10^{-10}\right)$. This procedure has been used in many previous studies (Tootell et al., 1997). A similar analysis was used to distinguish between positive and negative going MR fluctuations in the case of two-condition stimulus comparisons (MT mapping).

This analysis assumes that the noise is uncorrelated, an assumption known to be false for fMRI time series (Zarahn et al., 1997). The $p$ values reported should therefore be considered to be rough estimates of the levels of statistical significance of the periodic activation. However, the lack of any trace of activation in large stretches of nonretinotopic visual areas in the inferotemporal cortex and inferior parietal cortex suggests that this threshold is not too permissive.

The boundaries of retinotopic cortical areas (V1, V2, V3, VP, V3A, and $\mathrm{V} 4 \mathrm{v}$ ) were defined on the cortical surface for each individual subject on the basis of phase-encoded retinotopy (DeYoe et al., 1994, 1996; Engel et al., 1994, 1997; Sereno et al., 1995) and subsequent calculation of visual field sign, which provides an objective means of drawing borders between areas based on the angle between the gradients (directions of fastest rate of change) in the polar angle and eccentricity with respect to cortical position (Sereno et al., 1994, 1995). The visual field sign indicates whether each small patch of cortex represents the visual field as a mirrorimage or a nonmirror-image. As in nonhuman primates, early cortical areas (e.g., V1) are characterized by one visual field sign (e.g., mirrorimage). Adjacent areas often have an opposite visual field sign. Each field sign map shown here was based on at least four scans (two mapping polar angle and two mapping eccentricity).

The phase of the periodic response is delayed because of a finite vascular response time. Also, it is possibly differently delayed in different areas. In our stimuli, the basic stimulus frequency was low enough so that the hemodynamic delay was much smaller than one cycle, eliminating whole-cycle phase ambiguity. Data from a reversed-direction stimulus can be used to verify a map, but reversed data can also be combined with unreversed data to correct residual phase delay differences between areas (Sereno et al., 1995; Hagler and Sereno, 2006). In the $3 \mathrm{~T}$ data, we calculated the vector average at each voxel of the response amplitude and phase angle obtained for opposite directions of stimulus motion (clockwise versus counterclockwise) after reversing the sign of the phase angle for one direction. This procedure reduced noise in both visual and nonvisual cortical areas because the vector sum operation strongly penalizes voxels with inconsistent phase in opposite directions, even if they are separately significant.

To average mapping data across subjects, we used a new method for group analysis of phase-encoded retinotopic mapping data developed in our lab (Hagler and Sereno, 2006). The individual unfolded cortical surfaces were first inflated to a sphere, and then nonlinearly morphed into alignment with a canonical target sphere brain (icosahedral supertessellation) by minimizing local differences in average convexity ["sulcusness" (Fischl et al., 1999a, their Eq. 9)] while minimizing metric distortion. Complex-valued single-subject mapping data (amplitude and phase of significant periodic responses), were averaged across subjects by vector addition at each vertex of the canonical spherical surface. As with vector averaging of clockwise-counterclockwise data, this procedure strongly penalizes surface locations with inconsistent phase across subjects, even if those locations are significantly activated in each subject. The average map was then resampled back onto an individual brain for display. This averaging procedure was justified by the fact that surfacebased morphing does a good job of aligning independently obtained retinotopic maps (Fischl et al., 1999b).

To compare the position of human area V6 to what has been observed previously in the literature, we performed a statistical comparison between the average stereotaxic coordinates of the center of human area V6 as defined retinotopically in this study with the coordinates given by previous studies of putative V6 in the context of the V6 complex. We used a $t$ test for assessing differences between means, with a Bonferroni correction given that statistical parameters from the population are unknown and have to be estimated from sample statistics. We only included studies that used an approach similar to ours (i.e., a single-subject approach yielding a between-subjects mean and SD) because group data cannot be used for a $t$ test without between-subjects SD.

The macaque data were analyzed using CARET [computerized anatomical reconstruction and editing toolkit (Van Essen et al., 2001); http://brainmap.wustl.edu/caret/] to reconstruct and render threedimensional (3D) and two-dimensional representations of the right hemisphere of the case 16R reported by Galletti et al. (1999a) (Fig. 1E,F). Cells recorded from area $\mathrm{V} 6$ in case $16 \mathrm{R}$ were displayed on the $3 \mathrm{D}$ reconstruction of the brain to obtain location and extent of area V6. The cortical region containing cells with receptive field centers in the lower visual field was depicted in green, the region with receptive field centers in the upper visual field in red, and the region with receptive field centers 


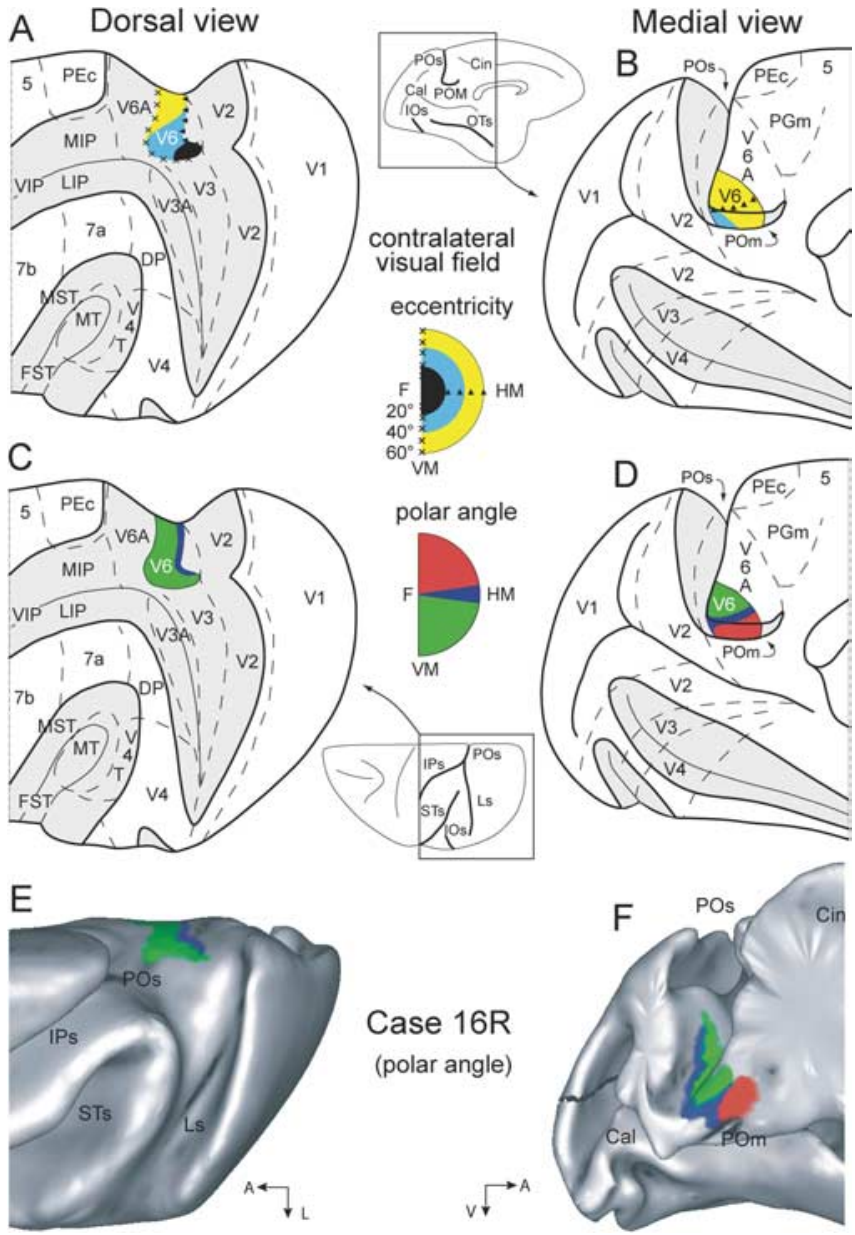

Figure 1. Topology and visual topography of macaque monkey area V6. $A-D$, Dorsal $(A, C)$ and medial $(\boldsymbol{B}, \boldsymbol{D})$ views of the left hemisphere of a macaque monkey brain (modified from Galletti et al., 1999a). P0, POM, inferior occipital (IOS), occipitotemporal (OTS), intraparietal (IPS), lunate (LS) and superior temporal (STS) sulci are opened to reveal areas in the depth of these sulci. Opened sulci are depicted in light gray, and are indicated as thickened lines on the brain silhouettes at the center of the figure. Dashed lines are the borders between different cortical areas, according to Colby et al. (1988), Desimone and Ungerleider (1986), Galletti et al. (1999a,b), Gattass et al. (1981, 1988), Gattass and Gross (1981), and Pandya and Seltzer (1982). In $\boldsymbol{A}$ and $\boldsymbol{B}$, the map of eccentricity in V6 is color-coded: central, parafoveal, and peripheral are represented as black, cyan, and yellow, respectively. $\boldsymbol{C}, \boldsymbol{D}$, The polar angle map of $\mathrm{V} 6$ is reported; lower, horizontal, and upper fields are represented as green, blue, and red, respectively. $\boldsymbol{E}, \boldsymbol{F}$, Dorsal and medial views of surface reconstructions of case 16R (Galletti et al., 1999a) performed using CARET software (see Materials and Methods). Polar angles of receptive fields recorded from V6 are indicated using the same color scale as in $\boldsymbol{C}$ and $\boldsymbol{D}$. HM, Horizontal meridian; VM, vertical meridian; DP, dorsal prelunate; VIP, ventral intraparietal; LIP, lateral intraparietal; MST, medial superior temporal; FST, fundus of the superior temporal; MIP, medial intraparietal.

on the horizontal meridian in blue. Reconstruction and registration procedures are reported in more detail in Galletti et al. (2005).

\section{Results}

\section{Polar angle representation}

Figures 2 and 3 show a color plot of the response to the rotating wedge stimulus, displayed on the flattened, folded, and inflated cortical surface representation from the left hemispheres of two participants. Color hue indicates the response phase, which is proportional to the polar angle of the local visual field representation. The flat maps in Figures 2 and 3 also show the boundaries of the early visual areas defined by mapping visual field sign (Sereno et al., 1995, 1994), and the location of the MT/medial superior temporal complex (labeled " $\mathrm{M}+$ ") mapped by addi- tional scans as described in Materials and Methods. In subject 1-SP (Fig. 2), the periphery was stimulated the most completely (up to $110^{\circ}$ total visual angle). The signal obtained is strong and consistent in all visual areas, and the functional activation extends from the occipital lobe up to the precuneate surface, to an extent not seen before. The greatly enlarged mapping stimuli used here revealed the presence of a previously unidentified upper field representation located near the peripheral lower field representations of areas V2 and V3. As indicated by the yellow square over the folded surfaces in Figures 2 and 3, this upper-field representation is not visible on a reconstruction of the pial surface of the brain (see 1-SP) because it is completely buried within the POS on the medial wall of the hemisphere. It is visible on the white-matter reconstruction of the brain and, even better, in inflated and flattened formats.

This previously unidentified upper field representation must belong to a previously unidentified visual area, because the upper-field representations of both areas V2 and V3 are located inferiorly, below the level of calcarine sulcus, and the known upper field representations of dorsal areas V3A and V7 (unlabeled region anterior to V3A) are located more laterally (Tootell et al., 1997, 1998). Because of the similarity of this region to macaque area V6 (see below), we labeled this area "human V6."

The bottom of Figure 3 shows the details of the polar angle representation in a series of eight close-ups of the flattened surface taken from the left hemisphere of one subject (2-GC). In each frame, a response-phase contour is marked with a white stripe and the complete range of phases in one hemifield $\left(180^{\circ}\right)$ is illustrated across the eight close-ups of the flattened surface. The phase contours communicate the shape of the retinotopic map more precisely to the viewer than the color map alone because small but significant variations in phase are hard to see with color alone. The phase contours show that phase varies systematically within the green, blue, and red regions (the color varies too, but the hue differences are small). In particular, the phase contours more precisely illustrate the position of visual field meridians (maxima or minima of polar angle near $-90,0$, and $90^{\circ}$ ), which are difficult to represent using hue alone.

The sequence of phase contours accurately represent the time sequence of activations for one stimulus cycle (although not the width of the activated band of cortex, which varies as a function of receptive field size and other factors). Starting in the lower field (green), the phase contours move apart (frames 1-2) indicating the presence of three vertical meridian borders: between $\mathrm{V} 1$ and V2, between V3 and V3A, and between V6 (yellow outline) and an unlabeled area superior and anterior (in unfolded view) to V6. This last contour then moves in an inferior and posterior direction (in frames 3-7), through the horizontal meridian (blue), finally joining a second contour at an upper vertical meridian representation in the center of the small medial upper field (red). A second joining of upper field contours is visible, as expected, at the border between V3A and V7, further laterally (lower left in flat map view). Thus, the borders of human V6 were defined by two vertical meridians, a more medial one over V2/V3 (in the middle of the red spot) and a more lateral one over V3A (in the middle of the green spot). Note that the entire new upper visual field representation (red spot) does not all belong to area V6, because in each subject tested (test and retest), the peak phase systematically moved apart in the middle of the red spot (Fig. 3, frame 8), indicating the presence of a vertical meridian. The doubling of the phase contour indicates that there is a second representation of the upper visual field outside V6.

The analysis of the polar angle data demonstrates the presence 
of a previously unidentified visual area, human V6, with a complete representation of the contralateral visual field along the polar angle axis (upper visual field $\rightarrow$ horizontal meridian $\rightarrow$ lower visual field). The location and extent of this area is indicated with a yellow outline in the first frame of the movie, as well as on the flattened and inflated surfaces of the brain. The upper field (red) of human V6 is located medially, just above area $\mathrm{V} 3$ and in front of dorsal area V2, whereas the lower field (green) is located superiorly and laterally, above areas V3/V3A. The horizontal meridian (blue) is located between the upper and lower fields, and is continuous with the horizontal meridian border between V2 and V3. This newly identified map is organized in a very similar way in the right hemisphere, and in both hemispheres of other subjects tested. Contralateral versus ipsilateral contrasts in voxels from human V6 reveal that the response to contralateral targets was highly significant ( $p=10^{-4}$ to $10^{-9}$ ), which matches the strictly contralateral representation in macaque V6 (Galletti et al., 1999a).

To verify the reliability of the retinotopic organization of human V6, we reversed the direction of rotation of the polar angle stimulus and compared the results obtained with opposite directions of movement within subjects. The stimulus always started at the bottom of the circle (at "6 o'clock"). In the first test, it rotated around the fixation point in a counter-clockwise direction, but in the second, it rotated in a clockwise direction, stimulating the four quadrants of the visual field in opposite order. Test 1 responses can therefore be used to predict test 2 responses with one free parameter, the hemodynamic delay (which is, however, small relative to the time for a full cycle of stimulus rotation). If the retinotopic organization of human V6 were stable and reliable, a reversed (CW) progression of the stimulus should elicit a response with a half-cycle delay, and with a reversed progression of phase. This is exactly what we found.

Figure 4 shows the results of the reversed (CW) stimulation on the left hemispheres of the same subjects illustrated in Figures 2 and 3. To make it easier to compare the results with those obtained in test 1 , we reversed the color map and added half a cycle of phase angle, so that upper, middle, and lower fields are colored the same for both directions of rotation. Note that maps of polar angle in area V6 were nearly identical in all subjects to those shown in Figures 2 and 3 , although they were acquired in independent experiments, performed months apart and with a reversed direction of ro-

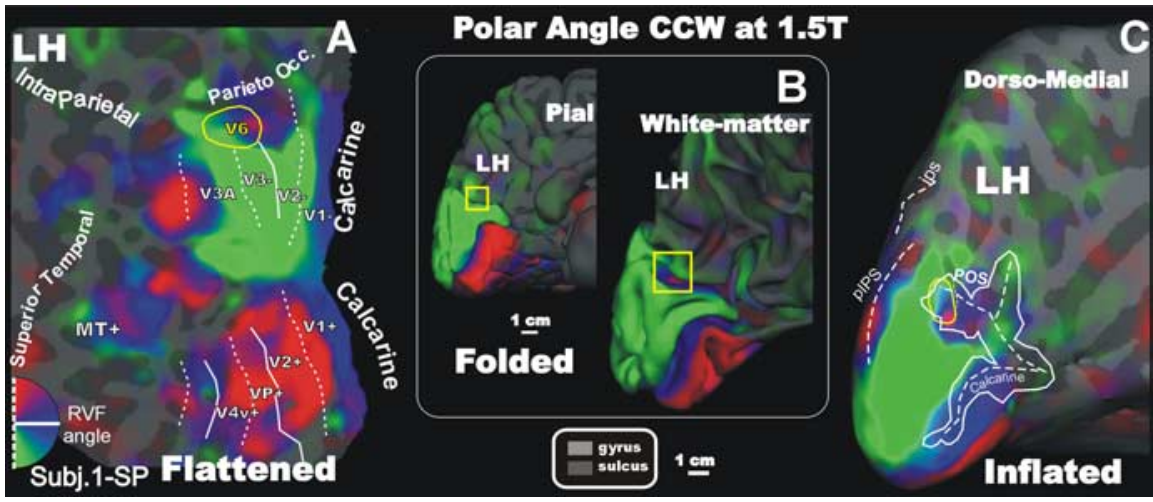

Figure 2. Retinotopy of polar angle representation of area V6 at 1.5T (CCW stimulus). Flattened ( $\boldsymbol{A})$, folded (B), and inflated (C) reconstructions of the left hemisphere (LH) of one participant (Subj.1-SP) are shown. The folded cortex (inside the white box and with its own scale bar) is shown in two versions: pial and white matter. Here and in all figures, yellow outlines indicate location (in folded) or borders (in flattened/inflated) of the human area V6. Red, blue, and green areas represent preference for upper, middle, and lower parts of the contralateral visual field, respectively. The newly identified dorsal area has a clear map of the contralateral hemifield. On the flat map, dotted and solid white lines indicate vertical and horizontal meridians. On the inflated map, the borders (closed lines) and fundi (dashed lines) of calcarine, intraparietal sulcus (IPS), and POS were estimated using two data sets generated during surface reconstruction and unfolding: (1) curvature, shown using light/dark gray to signify convex/concave (FreeSurfer "curv") and (2) summed movement perpendicular to vertices during inflation (FreeSurfer "sulc"). The scale bar $(1 \mathrm{~cm})$ on the bottom refers to the cortical surface of $\boldsymbol{A}$ and $\boldsymbol{C}$. RVF, Right visual field.
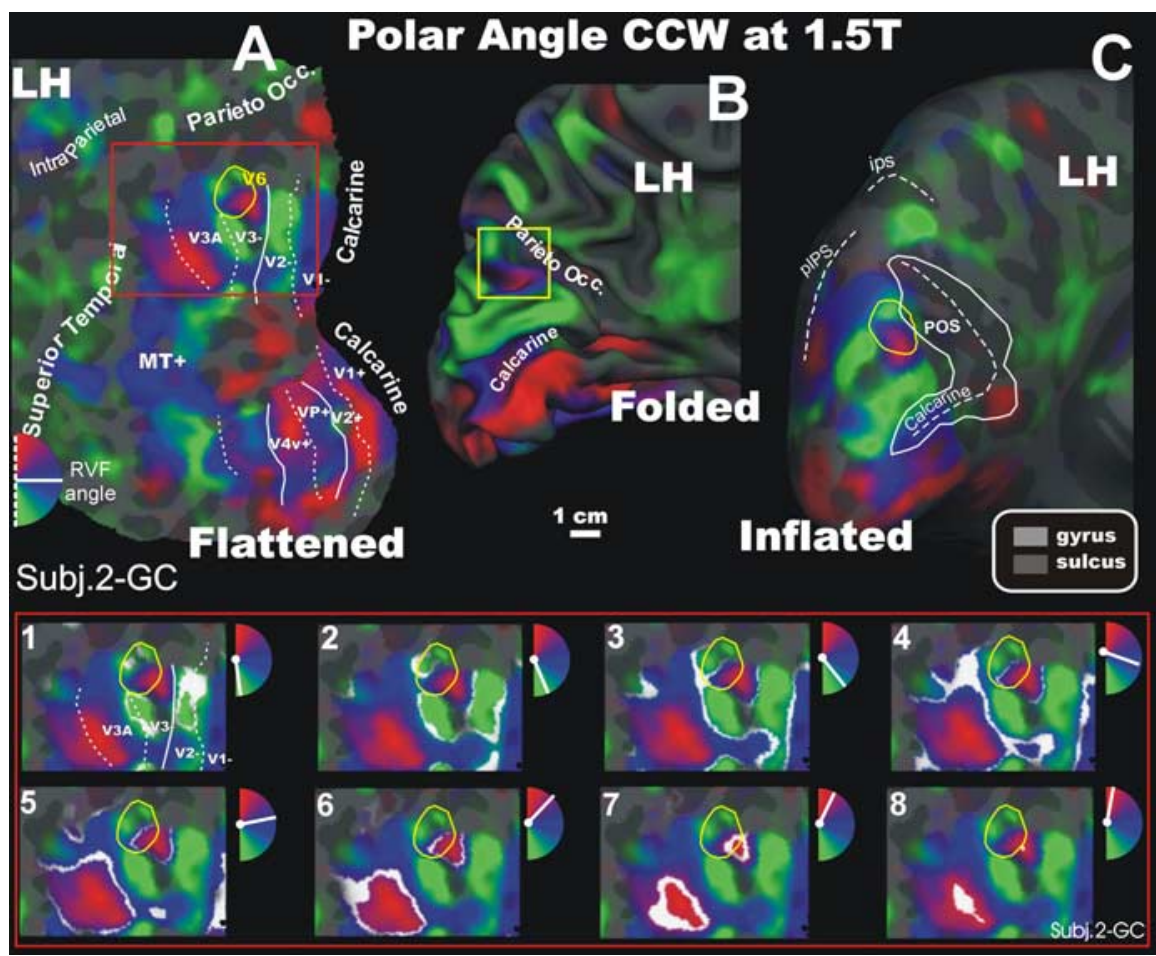

Figure 3. Retinotopy of polar angle representation of area V6 at 1.5T (CCW stimulus). Fattened (A), folded ( $\boldsymbol{B}$, white-matter version), and inflated ( $C$ reconstructions of the left hemisphere (LH) of another participant (Subj.2-GC) are shown. In the bottom part of the figure, a detailed organization of the polar angle representation is shown using close-up views of the flattened left hemisphere of the same subject (red box). The white areas on each panel represent the cortical regions activated by a single polar angle, indicated in white in the small hemifield icons located at the upper right of each snapshot. Other labels and logos are as in Figure 2. IPS, Intraparietal sulcus. RVF, Right visual field.

tation. The analysis of the phase contours (data not shown) confirmed for each subject that human area V6 contains a representation of the lower and upper visual fields, the former superior and anterior to $\mathrm{V} 3 / \mathrm{V} 3 \mathrm{~A}$, and the latter superior and anterior to $\mathrm{V} 2 / \mathrm{V} 3$.

Although data from a reversed-direction stimulus can be used 


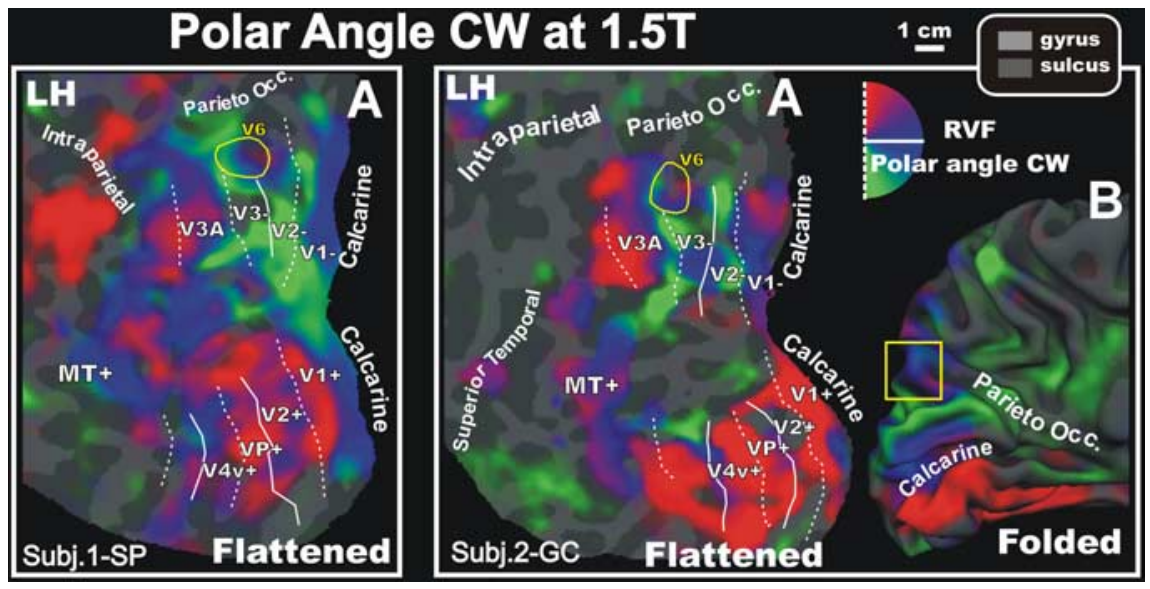

Figure 4. Retinotopy of $\mathrm{CW}$ polar angle representation of area $\mathrm{V} 6$ at 1.5T. Fattened $(\boldsymbol{A})$ and folded ( $\boldsymbol{B}$, white-matter version) reconstructions of the left hemisphere $(\mathrm{LH})$ of the same participants as in Figure 2 and 3 , but with the direction of the stimulation reversed from CCW to CW. The map color scheme for clockwise runs is reversed to make it consistent with counterclockwise runs shown in Figures 2-3. In both cases, red, blue, green areas represent preference for upper, middle and lower contralateral visual field, respectively. Other labels and conventions are as in Figure 2.

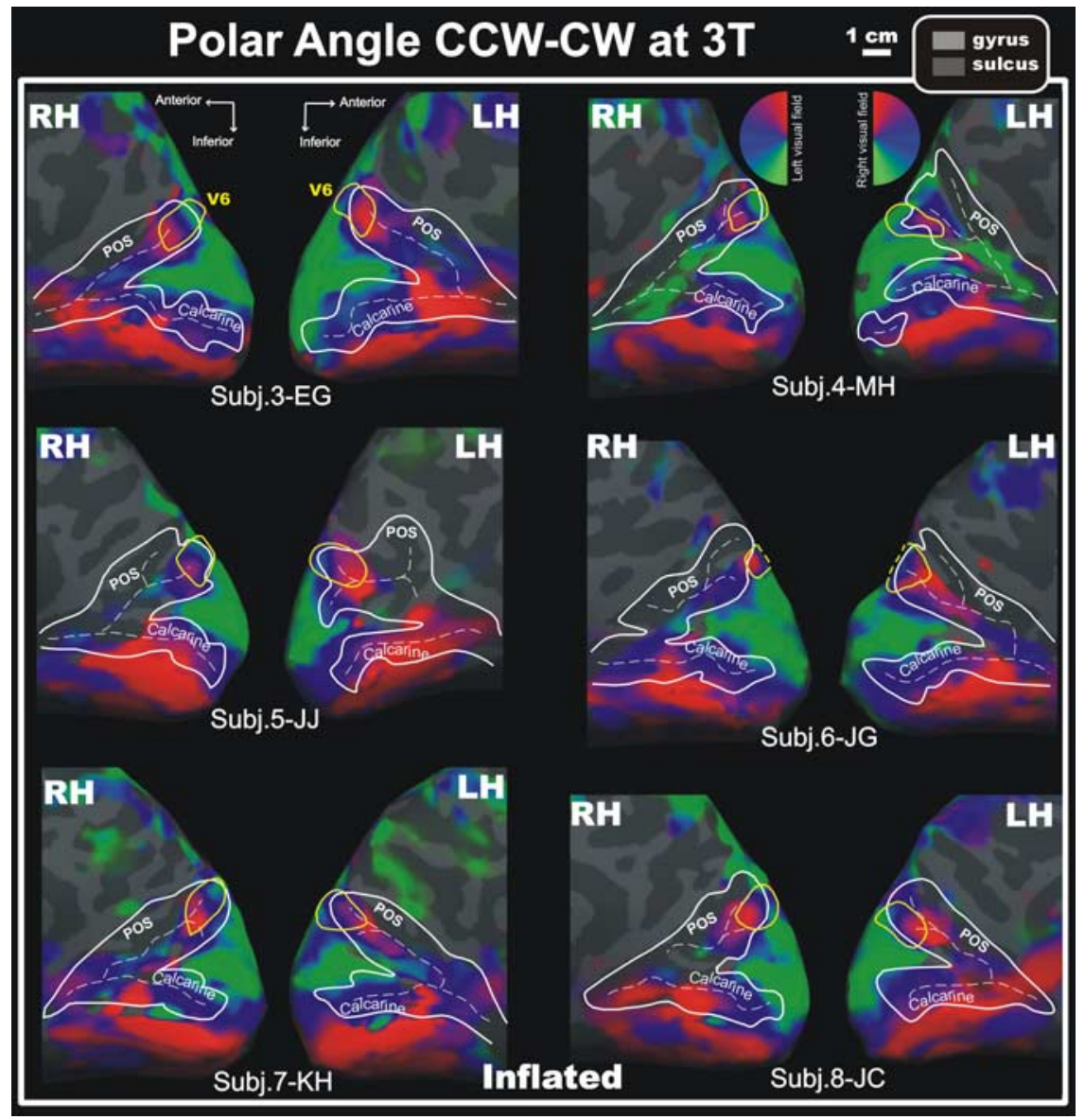

Figure 5. Consistent wide-field video retinotopy of the polar angle representation in area V6 by fMRI mapping at 3T. Results from both hemispheres [left hemisphere ( $\mathrm{LH})$; right hemisphere (RH)] of six additional participants scanned at $3 \mathrm{~T}$ are shown in medial inflated views. In this case, two $C \mathrm{CW}$ and two $\mathrm{CW}$ polar angle scans for each subject were combined to cancel hemodynamic delay differences among areas. We calculated the surface area of $V 6$ in the 3D surface for the 12 hemispheres: mean, $1.52 \mathrm{~cm}^{2} ; S D$, $0.22 \mathrm{~cm}^{2}$. Other labels and conventions are as in Figure 2.

to verify a map, reversed data can also be combined with unreversed data to correct residual phase delay differences. We applied this procedure to the $3 \mathrm{~T}$ data, which reduces noise in both visual and nonvisual cortical areas. Figure 5 shows the results of wide-field video retinotopy at 3T (complex-valued responses to $\mathrm{CW}$ and CCW stimuli were combined by vector addition after phase reversal (Sereno et al., 1995) (see Materials and Methods) in six (subjects 3-8) additional participants studied in a different laboratory, at a higher field strength. A small strictly contralateral representation of the entire hemifield (solid yellow outline) was visible in all 12 unfolded hemispheres in medial view, containing an upper field representation (red) on the medial surface completely separate from the more lateral one in V3A/V7 (not visible in this view), and a horizontal meridian (blue) and lower field representation (green) extending superiorly. In one participant, the lower field is just out of sight at the superior convexity of the unfolded surface (dashed yellow line). The activations found with video retinotopy precisely overlapped those revealed by standard checkerboards (data not shown). The similarity of these images to ones obtained with unidirectional data confirm that delay differences between areas were small relative to the full cycle time. In every case illustrated (6/6 left hemispheres and 6/6 right hemispheres), a similarly oriented hemifield representation was present in the posterior part of the superior end of the parieto-occipital sulcus. The same was true for 23 of 23 of the left and 23 of 23 of right hemispheres of the remaining $3 \mathrm{~T}$ subjects. In every hemisphere of every subject, the medial upper field representation in V6 was completely separated from the more lateral upper field in V3A/V7 by intervening lower fields.

We also performed a group analysis of phase-encoded polar angle data at 3T (for details, see Materials and Methods). Average results from 22 subjects (all 3 T subjects with two or more motion-artifact-free scans in each of the following conditions: counterclockwise polar angle, counterclockwise polar angle, outward eccentricity) are shown in Figure 6. The position and borders of area $\mathrm{V} 6$ are indicated with a dashed yellow outline. The location and retinotopic organization of the human area V6 was confirmed in the average map. Indeed the complete representation of the contralateral visual field, as well as the location of the upper-field representation medial and inferior to the lower field, closely parallel the individual data (Figs. 2-5).

\section{Eccentricity representation}

We also mapped eccentricity in human V6. Figure 7 shows a color plot of the response to a wide-field ring stimulus expanding at a 


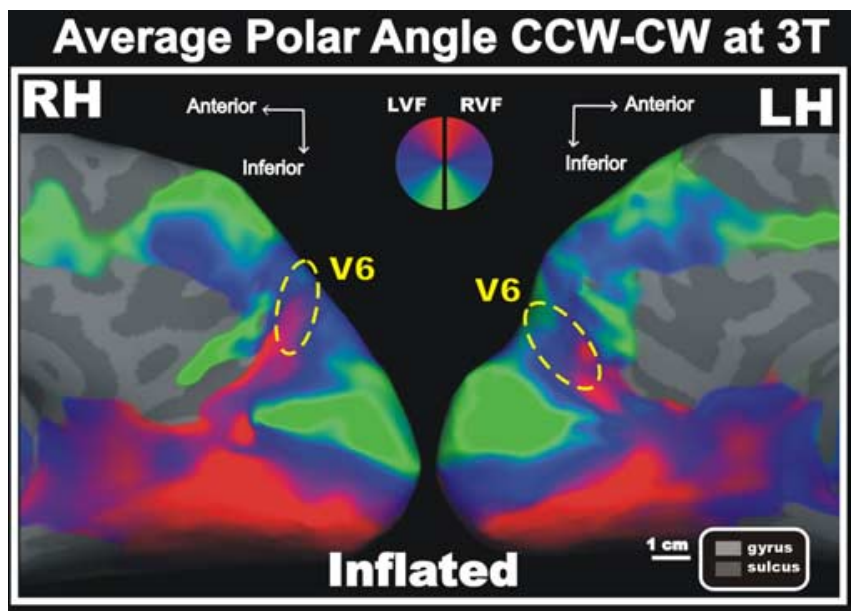

Figure 6. Average polar angle data. 3T average phase-encoded polar angle maps $(n=22$ subjects) rendered on close-up views of the inflated left $(\mathrm{LH})$ and right $(\mathrm{RH})$ cortical hemispheres are shown. Here, polar angle maps show the average of $2-12$ scans acquired over one to three sessions. Also in the average map, CCW and CW polar angle scans were combined in each subject to cancel hemodynamic delay differences among areas. Dashed yellow ellipsoids indicate the position of the human area V6. Also in the average map, the presence of an extra upper-field representation on the medial wall is clearly appreciable. We calculated the average retinotopy of the V6 area (average of left and right hemisphere ellipses): mean, $2.1 \mathrm{~cm}^{2}$. The area of $\mathrm{V} 6$ estimated from the retinotopic average is likely to be a slight overestimate as a result of blurring caused by intersubject differences. Other labels and logos are as in Figure 2 .

constant slow speed $(\sim 1 \%)$, displayed on both hemispheres of three participants. The hue of the color at each cortical surface point again indicates the response phase, but now it is proportional to the eccentricity of the local visual field representation. The outline of putative area V6 of each subject, as obtained by polar angle mapping, has been drawn on the eccentricity map to aid comparisons. Eccentricity increases as one moves inferiorly on the inflated mesial surface. Note that moving inferiorly on the inflated surface corresponds to a horizontal movement toward the calcarine incision on the flat maps. In all subjects, the putative area V6 contains a central representation of the visual field laterally (and slightly superiorly), and a peripheral representation of the visual field medially. This is in line with the macaque data (Fig. 1), where the representation of the central $20^{\circ}$ of the visual field is located at the lateral end of POS, and the most peripheral representation $\left(>40^{\circ}\right)$ is at the medial end of POS and on the mesial surface of the hemisphere.

The detailed organization of the representation of eccentricity in the left and right hemisphere of one subject (1-SP) is also shown as a sequence of four close-ups of the flattened surface in Figure 8 . The analysis of isoeccentricity contours reveals the presence of a foveal representation in the most lateral part of V6 (indicated with an asterisk in the figures). The location of foveal representation in V6 stands apart from the foveal representations of areas V2 and V3, and also from the foveal representation of $\mathrm{V} 3 \mathrm{~A}$, shown previously to be separated from foveal V2 and V3 (Tootell et al., 1997).

Data from Figures $2-8$ show that by using wide-field visual stimuli, there is no reversal of activation phase in the far peripheral representations of areas V1 and V2, which is sometimes seen with less extensive visual mapping stimuli (Sereno et al., 1995). Those reversals are likely mapping artifacts caused by surround inhibition elicited in the far peripheral parts of $\mathrm{V} 1$ and $\mathrm{V} 2$ beyond the maximum eccentricity of the stimulus (Sereno and Tootell, 2005); this mapping artifact is greatly reduced by using wide field stimuli.
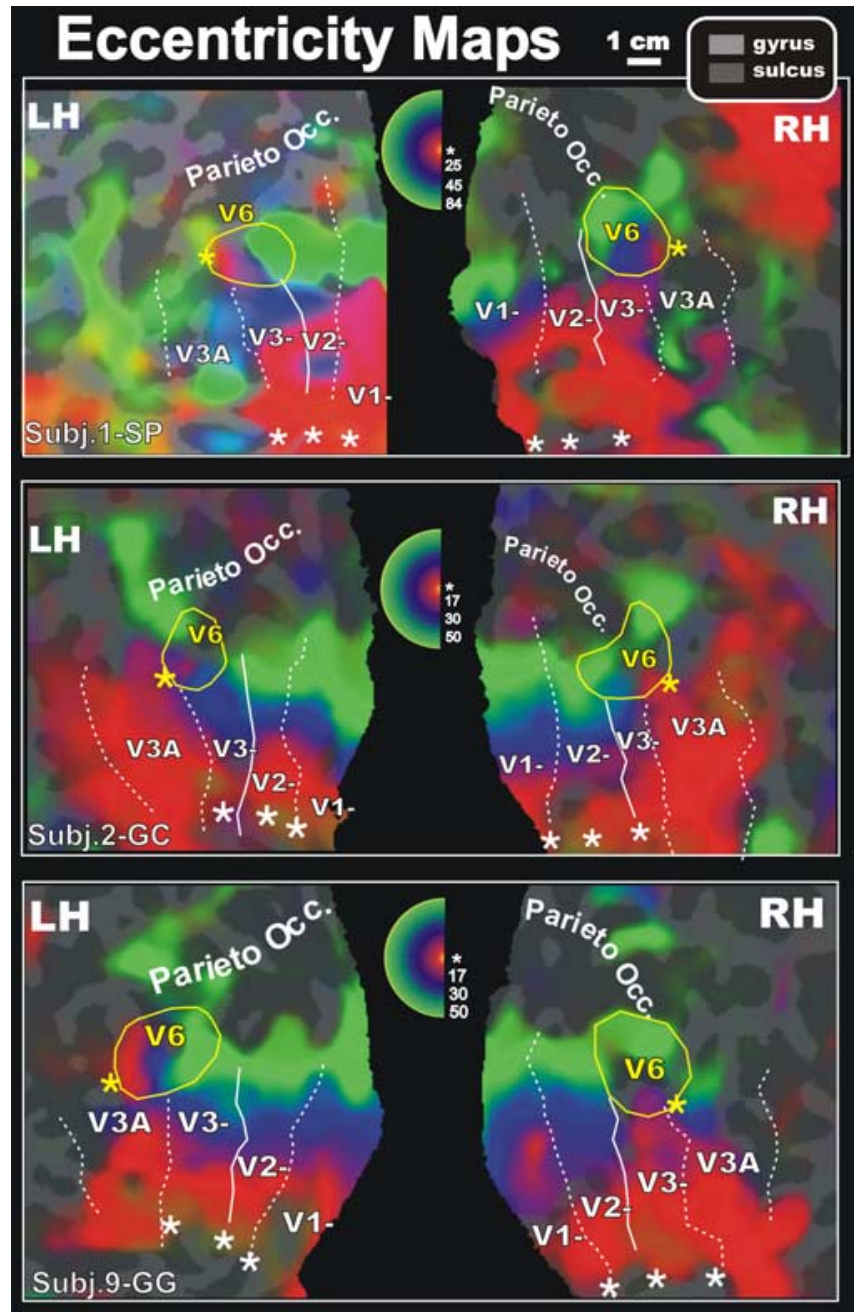

Figure 7. Retinotopy of eccentricity representation of area V6 by fMRI mapping at $1.5 \mathrm{~T}$. Phase-encoded eccentricity maps rendered on close-up views of the left (LH) and right (RH) flattened hemispheres in the same participants as in Figures 2 and 3 and in a new participant (Subj.9-GG). The representation of central-through-more-peripheral eccentricities is coded using red-blue-green, respectively (see pseudocolor inset, located in between the left and right hemispheres of each participant). The eccentricity map of Subj.1-SP (left hemisphere) also shows some yellow in a few cortical locations. Unlike polar angle maps, eccentricity maps "wrap around:" there is a narrow band of phases that are ambiguous between extreme central eccentricities and extreme peripheral eccentricities. The exact wrap-around point is coded in yellow (between red and green). Each inset indicates the maximal periphery we were able to reach in that subject. The representations of the center of gaze are indicated with asterisks. Area V6 clearly has its own representation of the fovea, distinct from the foveal representation of the other dorsal visual areas.

Figure 9 shows a map of the visual field sign (yellow, mirror image of visual field; blue, nonmirror image of visual field) on the flattened surface of the left hemispheres of two participants (1-SP and 2-GC), calculated from the maps of polar angle and eccentricity from the same subjects, as described previously (Sereno et al., 1995). The area we have defined as human V6 contains a mirror-image representation located just above V3 and V3A, and is present bilaterally in all subjects.

\section{Brain location}

As in macaque, human V6 is generally located within the POS. The most superior part of the human POS often shows a "Y" shape, with anterior and posterior branches variably convoluted across individuals. Although there was some small variation in 


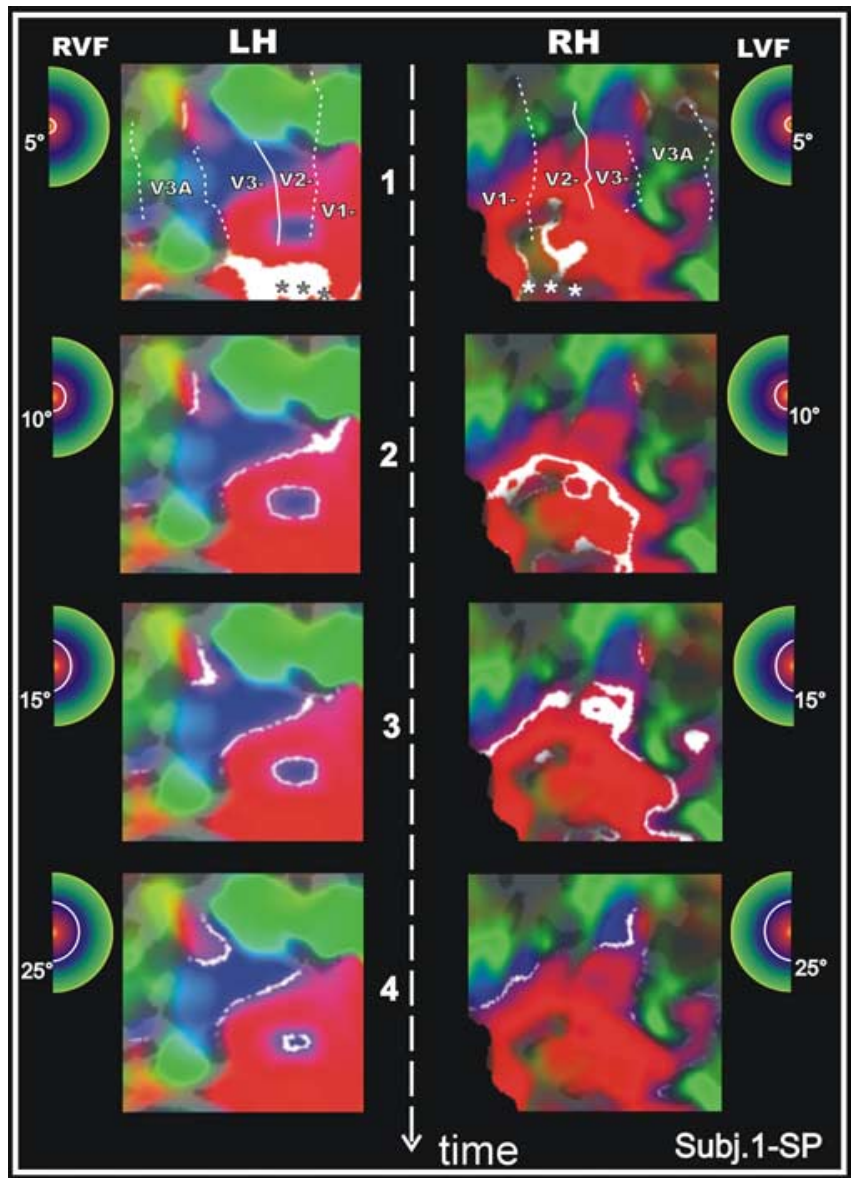

Figure 8. Phase-encoded retinotopy of eccentricity representation of area V6. The eight panels show a close-up map of eccentricity in V6 in the left ( $\mathrm{LH}$; first column) and right ( $\mathrm{RH}_{\text {; }}$ second column) hemispheres of one subject (1-SP). The white areas on each panel represent the cortical regions activated by eccentricities indicated in white in the small hemifield icons located next to each panel. Only the central $25^{\circ}$ of eccentricity are shown in four steps in the direction indicated by the dashed white arrow. RVF, Right visual field; LVF, left visual field.

the exact form and position of the V6 map across subjects, it turned out to be reliably located in or near the posterior branch of the dorsal end of the POS (Figs. 2-8). In conventional MNI coordinates, area V6 (as defined retinotopically in this study), was centered at $x=-11 \pm 7, y=-77 \pm 5, z=46 \pm 14$. Talairach coordinates were very similar $(x=-11 \pm 7 ; y=-72 \pm 4 ; z=46$ \pm 13 ), the only slight difference being in the $y$-axis (Talairach and Tournoux, 1988). Note that when plotted on the representative single subject provided by SPM, the group stereotaxic coordinates are located in the anterior (instead of the posterior) branch of the POS. This difference with the individual maps may be because of peculiarities of the SPM single subject in this region, as well as to the intrinsic inaccuracy of the normalization procedures. In any case, the expected sulcal location of V6 (MNI coordinates: $x=-9, y=-82, z=36$; Talairach coordinates: $x=-9$, $y=-78, z=37)$ on the representative SPM brain $(x=-9 ; y=$ $-82 ; z=36)$ is less than one SD away from our group mean coordinates.

Average Talairach coordinates were also calculated for area V3A $(x=-18 \pm 5 ; y=-78 \pm 3 ; z=25 \pm 3)$. They fit well with the coordinates given by Tootell et al. (1997) for the superior medial end of their V3A area $(x= \pm 14 ; y=-84 ; z=19)$, which in turns agrees with the fact that our stimuli likely more fully activated the peripheral field representation in $\mathrm{V} 3 \mathrm{~A}$, which occu-

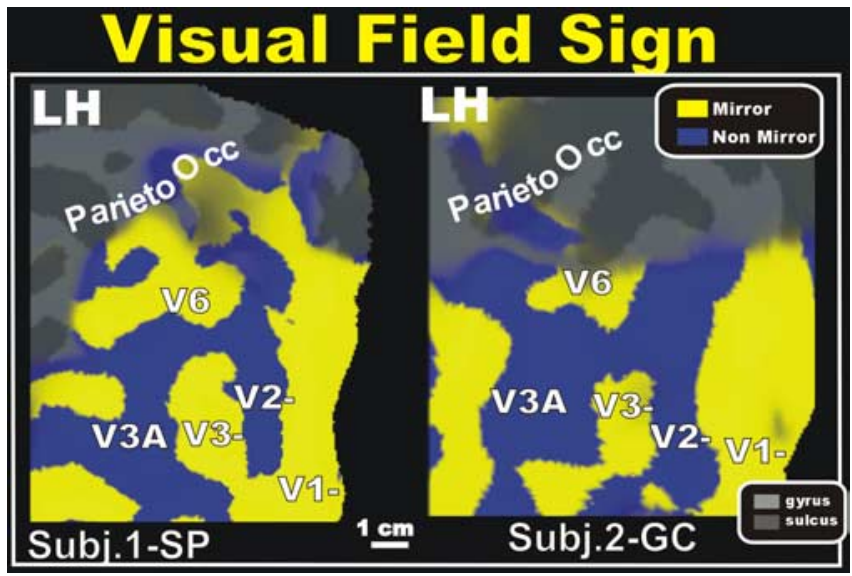

Figure 9. Maps of retinotopic field sign in area V6. Analysis of retinotopic data (polar angle and eccentricity) by visual field sign (mirror-image versus nonmirror-image visual field representation) (Sereno et al., 1995). Mirror-image areas (yellow; e.g., V1), and nonmirror-image areas (blue; e.g., V2) are shown in the left (LH) flattened hemispheres of two participants (1-SP; 2-GC). The lower field representations of V1, V2, V3, and V3A are labeled, along with V6. Note that $\mathrm{V} 6$ contains a mirror-image representation, like V1 and V3, and opposite to V2 and V3A.

pies the superior medial end of V3A. Our putative human V6 is slightly anterior, superior, and medial to V3A. The distance between V3A and V6 together with the duplication of the phase contours (indicating that there are two representations of the same part of the visual field) militate against an interpretation of V6 as a peripheral portion of area V3A. As suggested by Sereno et al. (2001), it is possible that human V3A as originally defined may have covered somewhat more than one visual area. Interestingly, macaque V3A has also been subdivided. The original definition of macaque V3A suggested that it might contain two areas, a medial and a lateral one (Van Essen and Zeki, 1978); the medial area, with larger receptive field sizes, may have partly overlapped V6 (Galletti et al., 1999a).

The discovery of a previously unidentified extrastriate area extends our notion of the basic set of early visual areas in humans. Figure 10 shows the location and topography of the newly identified cortical area V6 together with other early visual areas mapped with wide-field retinotopic stimuli. As shown in the figure, the wide-field retinotopic stimuli used in the present study also revealed the actual size of striate and extrastriate areas, which look larger than in previous brain mapping studies. Activation extends from the occipital lobe up to the precuneate surface, to an extent not seen before with standard mapping stimuli.

The wide-field retinotopic data here also provided preliminary evidence for the existence of two additional areas. First, medial and inferior to the human V6, we consistently found an additional small upper-field-only representation (not labeled) that bordered the upper-field representation of V6. In each subject, starting from the upper field, the phase contour splits and moves apart in the middle of the upper-field representation (see red spot in frame 8 of Fig. 3) indicating the presence of a second representation of the upper visual field. This additional upper field map was organized in a similar way across hemispheres and subjects. It was dominated by the periphery, with no obvious foveal representation, and it seemed to contain a nonmirrorimage representation of the visual field (Fig. 9). Second, anterior and lateral to V6, is a region with a small ipsilateral versus contralateral difference and inconsistent polar angle signals across subjects and sessions. The eccentricity maps showed that this area also represents the extreme periphery, typically starting to respond at over $35^{\circ}$ eccentricity. 


\section{Discussion}

Retinotopic mapping with fMRI has been used to identify human homologues of areas originally described in nonhuman primates (Sereno et al., 1995; Tootell et al., 1997). Here, we mapped the organization of human visual area V6, located in the posterior parieto-occipital sulcus using wide-field retinotopic stimulation. In 34 subjects tested, we found a map of the contralateral hemifield containing a characteristic medially located upper-field representation separated from the one in V3A, just anterior to peripheral V2/V3 lower representations. The V6 lower field is superior to its upper field on the unfolded cortex, anteromedial to peripheral V3/V3A. The organization and neighbor relations of this area closely resemble those reported for macaque V6 (Fig. 1) (Galletti et al., 1999a).

Human V6 has a representation of the center of gaze separate from the foveal representations of $\mathrm{V} 1 / \mathrm{V} 2 / \mathrm{V} 3$. Eccentricity plots suggest that central and peripheral visual field representations have similar extents, as with macaque V6 (Fig. 1) (Galletti et al., 1999a). This contrasts with central overrepresentation in $\mathrm{V} 1 / \mathrm{V} 2 / \mathrm{V} 3 / \mathrm{V} 3 \mathrm{~A}$. A similar situation was previously found in owl monkey area $\mathrm{M}$, located on the mesial surface of the occipital lobe (Allman and Kaas, 1976), in a position similar to macaque V6.

Visual field sign calculations (Fig. 9) show that V6 has a mirror-image representation, like V1 and V3.

\section{Comparison with previous human studies}

Dechent and Frahm (2003) suggested the existence of a human V6-complex (V6+V6A) with a less-emphasized foveal representation, as in macaque monkeys. However, macaque V6 and V6A have different functional properties, and V6A is not obviously retinotopic (Galletti et al., 1999a, 1999b). In contrast, human V6 and V6A in those studies were distinguished only as the inferior and superior parts of a POS focus instead of being based on functional criteria.

The V6 complex (De Jong et al., 2001; Simon et al., 2002; Dechent and Frahm, 2003) has average Talairach coordinates $x=$ $22 \pm 3, y=-64 \pm 12$, and $z=13 \pm 10$, which differ from our retinotopically defined V6 (Talairach coordinates: $x=-11 \pm 7$, $y=-72 \pm 4, z=46 \pm 13$ ), especially in the $z$-axis. A direct statistical comparison could be performed for one study (Dechent and Frahm, 2003); it showed a significant difference both in the $y$ - $(t=3.8 ; p<0.01)$ and $z$-axis $(t=6.14, p<0.016)$ $(x$-axis data were not reported). Our mean V6 Talairach coordinates correspond better to those given in previous studies for V6A $(x=8 \pm 9 ; y=-66 \pm 3 ; z=31 \pm 17)$ than for V6. Cheng et al. (1995) activated foci (3, 4 in their Tables 1,2$)$ near our V6 using coherent or incoherent movement of random dot fields.

\section{Brain location of human V6}

V6 is located within the POS in both humans and macaques. However, the folded reconstructions of the medial surface (Fig. 11 , left) show that human V6 is superior to macaque V6. Other visual areas occupy different locations in human and macaque brains as a consequence of the expansion of laterally placed nonprimary areas (e.g., the central representation of $\mathrm{V} 1$ is displaced posteriorly, area LIP + superiorly, and MT/V5 inferiorly). The relatively more superior position of human V6 appears to counter this trend, as one might have expected the superior-tomedial movement of human parietal visual areas to have pushed V6 further inferior on the medial wall. This apparent paradox can be resolved by considering how $\mathrm{V} 1$ has changed with respect to the calcarine in humans. The posterior-around-to-medial movement of human V1 has two effects: (1) the superior/inferior extent of V1 is reduced at the occipital pole because the V1 central representation $\left(7^{\circ}\right)$, which forms a lateral occipital operculum in macaques, moves into the posterior calcarine, and (2) the peripheries of V1 and V2 are pushed anteriorly, overflowing the calcarine cortex onto the medial wall (Fig. 11). V6 sits in a more superior position in humans because it is no longer overlaid by an occipital operculum and because of the peripheral V1/V2 "overflow." Nevertheless, its neighbor relations on the unfolded surface remain essentially unchanged.

\section{Homologies of dorsal and medial occipital areas among primates}

Dorsal and medial visual areas were first mapped in detail in smooth-brained New World monkeys. Third-tier extrastriate areas were recognized by the presence of upper visual fields near dorsal, lower-field-only V2. Allman and Kaas' (1975) area DM (dorsomedial) had dorsolateral upper fields and dorsomedial lower fields. Allman and Kaas (1976) mapped a second area, M, completely confined to the medial wall. Area $\mathrm{M}$ had the greatest deemphasis of the center of gaze of any extrastriate area, and was more responsive to moving random dot fields than DM (Baker et 


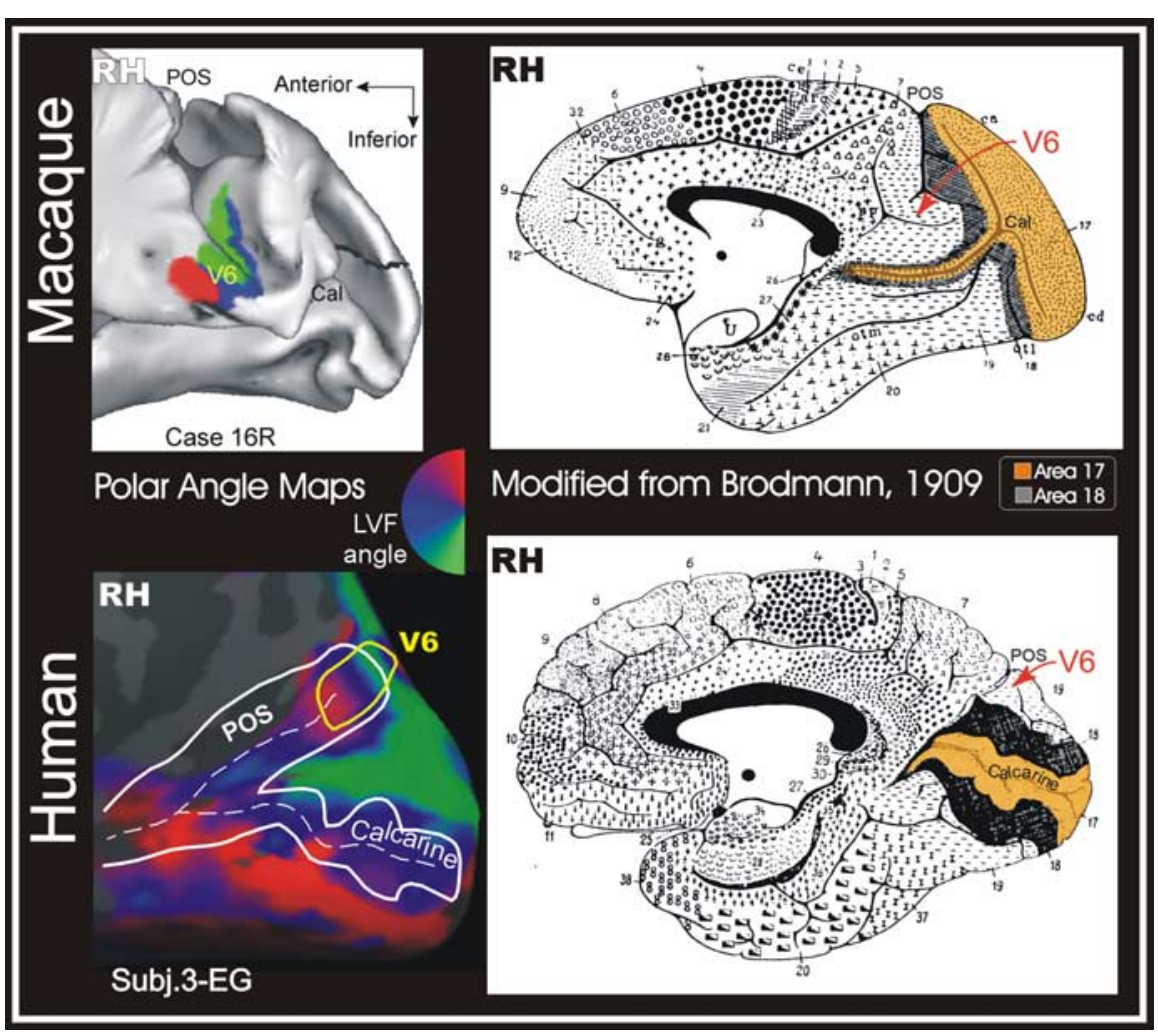

Figure 11. V6 location in macaque and human brains. Left, Retinotopic maps of polar angle representation of area V6 in macaque (top) and human (bottom) brains. Polar angle maps are rendered on the folded (top) and inflated (bottom) cortical surface reconstructions of the caudal part of the right hemisphere (RH) of the macaque [case 16R from Galletti et al., (1999a)] and one human subject (Subj.3-EG; 3T data), respectively. Both macaque and human retinotopic data show a clear and similarly arranged map of the contralateral left hemifield (red, upper left visual field; blue, left horizontal; green, lower left). Right, Medial views of macaque (top) and human (bottom) brains (right hemisphere), showing the Brodmann's parcellation of the cortical surface (Brodmann, 1909). Areas 17 and 18 are color coded (orange and dark gray, respectively). The location of area V6 is indicated by a red arrow on both brains. Labels and conventions are as in Figures 1 and 2.

al., 1981). Upper field $M$ was completely separated from upper field DM by lower fields (of DM and M).

In the larger, more folded cortex of macaque monkeys, a dorsal area containing upper visual fields, V3A, was originally described by Zeki (1978). Unlike owl monkey DM, which touches V2 (Allman and Kaas, 1975; Sereno et al., 1994; cf. Lyon et al., 2002), V3A was separated from V2 by a thin, lower-field-only area, V3. Gattass et al. (1986) subsequently described a second, medially located, upper-field-containing area, PO, which, like owl monkey area $\mathrm{M}$, contained upper fields distinct from those in laterally placed V3A. Thus, two separated clusters of dorsally located upper fields characterize both $\mathrm{New}$ and Old World monkeys.

Subsequent work on New World cebus monkeys (Neuenschwander et al., 1994) recognized PO (touching V2, upper fields medial), and POd (touching PO, upper fields medial). Rosa and Schmid (1995) mapped the dorsal cortex in the New World marmoset and presented a new scheme for area DM where all but central upper fields are represented as a continuous mirrorimage patch [like Allman and Kaas' (1975) original DM] mostly touching V2, whereas central upper fields of this DM are appended laterally (along a discontinuity) as a nonmirror-image patch. A far medial upper-field-only medial PO (POm) was adjoined anteriorly by an area $\mathrm{M}$ that did not touch V2. Recently, Rosa and Tweedale $(2001,2005)$ suggested that Old World monkeys might be similarly organized: most of upper field V3A is taken as the lateral (nonmirror-image) DM upper field, whereas PO is subsumed into the dorsal, mirror-image part of a marmoset-like DM. In this scheme, DM is a synonym for V6.

Returning to macaques, Galletti et al. (1999a,b, 2005) described a posterior V6 extending posterior to $\mathrm{PO}$ of Colby et al. (1988), and an anterior V6A extending anterior to it. Lewis and Van Essen (2000), in contrast, described a large $\mathrm{PO}$ touching V2 medially but extending away from it moving laterally, the opposite of Rosa's DM/V6 (Rosa and Tweedale, 2005), which extends away from V2 moving medially. Finally, Lewis and Van Essen (2000), Colby et al. (1988), and Gattass et al. (2005) recognized an additional area, posterior intraparietal, between PO and V3A.

The somewhat inconsistent parcellations arise from the fact that upper (or lower) visual field representations are often adjoined by another upper (or lower) field belonging to a different area; for example, lower field V1/V2, upper field V1/ V2, lower field MT/V4t, and upper field $\mathrm{MT} /$ fundus of the superior temporal all touch. An obvious cytoarchitectonic border distinguishes V1/V2, but most other interareal borders are less clear, and it is difficult to collect electrophysiological mapping data that are both dense and extensive, especially if one pauses to measure functional properties. Although the spatial resolution of fMRI is not as high as electrophysiology, it is more uniform. We have used this to our advantage to generate a testable proposal for the retinotopic organization of human V6 (cf. Koyama et al., 2004; Sereno and Tootell, 2005; Orban et al., 2006).

\section{Surrounding areas}

We found two regions surrounding V6: a small medial upperfield-only representation and an anterolateral region with variable retinotopic organization. A medial upper-field-only representation bordering V2 was described in marmosets (area POm) (Rosa and Schmid, 1995), but not (yet) in macaques. The region anterolateral to human V6 may be a homolog of macaque V6A given its location and vertical meridian border with V6; as in macaque V6A (Galletti et al., 1999b), it lacks repeatable contralateral retinotopic organization. More studies are needed to characterize these areas in humans.

\section{Conclusions}

A newly recognized extrastriate area in humans, V6, was mapped in 34 subjects. This definition improves our understanding of early visual areas. Mapping this area required the use of extremely wide-field stimuli more closely resembling natural stimulation, a method that has proved useful for mapping other visual areas with well developed peripheral representations (Sereno et al., 2004, 2005). Preliminary data show that human V6 is selectively activated by coherent motion of random dot fields (Pitzalis et al., 2005), similar to macaque V6 (C. Galletti, unpublished observa- 
tions) and owl monkey area M (Baker et al., 1981). Although cortical area homologies are often controversial (Kaas, 2003; Rosa and Tweedale, 2004), the similarity in position, peripheral emphasis, internal organization, and functional properties argue that this area is the human homolog of macaque area V6. Similarities with owl monkey area $\mathrm{M}$ suggest that an area similar to human V6 may characterize all anthropoids.

\section{References}

Allman JM, Kaas JH (1975) The dorsomedial cortical visual area: a third tier area in the occipital lobe of the owl monkey (Aotus trivirgatus). Brain Res 100:473-487.

Allman JM, Kaas JH (1976) Representation of the visual field on the medical wall of occipital-parietal cortex in the owl monkey. Science 191:572-575.

Baker JF, Petersen SE, Newsome WT, Allman JM (1981) Visual response properties of neurons in four extrastriate visual areas of the owl monkey (Aotus trivirgatus): a quantitative comparison of medial, dorsomedial, dorsolateral, and middle temporal areas. J Neurophysiol 45:397-416.

Brett M, Johnsrude IS, Owen AM (2002) The problem of functional localization in the human brain. Nat Rev Neurosci 3:243-249.

Brewer AA, Press WA, Logothetis NK, Wandell BA (2002) Visual areas in macaque cortex measured using functional magnetic resonance imaging. J Neurosci 22:10416-10426.

Brodmann K (1909) Vergleichende lokalisationslehre der Großhirnrinde. Leipzig, Germany: Barth.

Cheng K, Fujita H, Kanno I, Miura S, Tanaka K (1995) Human cortical regions activated by wide-field visual motion: an H2(15)0 PET study. J Neurophysiol 74:413-427.

Colby CL, Gattass R, Olson CR, Gross CG (1988) Topographycal organization of cortical afferents to extrastriate visual area PO in the macaque: a dual tracer study. J Comp Neurol 269:392-413.

Dale AM, Fischl B, Sereno MI (1999) Cortical surface-based analysis. I. Segmentation and surface reconstruction. NeuroImage 9:179-194.

Dechent P, Frahm J (2003) Characterization of the human visual V6 complex by functional magnetic resonance imaging. Eur J Neurosci 17:2201-2211.

De Jong BM, van der Graaf FH, Paans AM (2001) Brain activation related to the representations of external space and body scheme in visuomotor control. NeuroImage 14:1128-1135.

Desimone R, Ungerleider LG (1986) Multiple visual areas in the caudal superior temporal sulcus of the macaque. J Comp Neurol 248:164-189.

DeYoe EA, Bandettini P, Neitz J, Miller D, Winans P (1994) Functional magnetic resonance imaging (FMRI) of the human brain. J Neurosci Methods 54:171-187.

DeYoe EA, Carman GJ, Bandettini P, Glickman S, Wieser J, Cox R, Miller D, Neitz J (1996) Mapping striate and extrastriate visual areas in human cerebral cortex. Proc Natl Acad Sci USA 93:2382-2386.

Engel SA, Rumelhart DE, Wandell BA, Lee AT, Glover GH, Chichilnisky EJ, Shadlen MN (1994) fMRI of human visual cortex. Nature 369:525.

Engel SA, Glover GH, Wandell BA (1997) Retinotopic organization in human visual cortex and the spatial precision of functional MRI. Cereb Cortex 7:181-192.

Felleman DJ, Van Essen DC (1991) Distributed hierarchical processing in the primate cerebral cortex. Cereb Cortex 1:1-47.

Fischl B, Sereno MI, Dale AM (1999a) Cortical surface-based analysis. II. Inflation, flattening, and a surface-based coordinate system. NeuroImage 9:195-207.

Fischl B, Sereno MI, Tootell RB, Dale AM (1999b) High-resolution intersubject averaging and a coordinate system for the cortical surface. Hum Brain Mapp 8:272-284.

Friston KJ, Ashburner J, Poline JB, Frith CD, Heather JD, Frackowiak RSJ (1995) Spatial registration and normalization of images. Hum Brain Mapp 2:165-189.

Galletti C, Fattori P, Battaglini PP, Shipp S, Zeki S (1996) Functional demarcation of a border between areas V6 and V6A in the superior parietal gyrus of the macaque monkey. Eur J Neurosci 8:30-52.

Galletti C, Fattori P, Gamberini M, Kutz DF (1999a) The cortical visual area V6: brain location and visual topography. Eur J Neurosci 11:3922-3936.

Galletti C, Fattori P, Kutz DF, Gamberini M (1999b) Brain location and visual topography of cortical area V6A in the macaque monkey. Eur J Neurosci 11:575-582.
Galletti C, Gamberini M, Kutz DF, Fattori P, Luppino G, Matelli M (2001) The cortical connections of area V6: an occipito-parietal network processing visual information. Eur J Neurosci 13:1572-1588.

Galletti C, Gamberini M, Kutz DF, Baldinotti I, Fattori P (2005) The relationship between V6 and PO in macaque extrastriate cortex. Eur J Neurosci 21:959-970.

Gattass R, Gross CG (1981) Visual topography of striate projection zone (MT) in posterior superior temporal sulcus of the macaque. J Neurophysiol 46:621-638.

Gattass R, Gross CG, Sandell JH (1981) Visual topography of V2 in the macaque. J Comp Neurol 201:519-539.

Gattass R, Sousa APB, Covey E (1986) Cortical visual areas of the macaque: possible substrates for pattern recognition mechanisms. Exp Brain Res [Suppl] 11:1-20.

Gattass R, Sousa APB, Gross CG (1988) Visuotopic organization and extent of V3 and V4 of the macaque. J Neurosci 8:1831-1845.

Gattass R, Nascimento-Silva S, Soares JG, Lima B, Jansen AK, Diogo AC, Farias MF, Botelho MM, Mariani OS, Azzi J, Fiorani M (2005) Cortical visual areas in monkeys: location, topography, connections, columns, plasticity and cortical dynamics. Philos Trans R Soc Lond B Biol Sci 360:709-731.

Hagler Jr DJ, Sereno MI (2006) Spatial maps in frontal and prefrontal cortex. NeuroImage 29:567-577.

Kaas JH (2003) Early visual areas: V1, V2, V3, DM, DL, and MT. In: The primate visual system (Kaas JH, Collins CE, eds), pp 139-159. Boca Raton, FL: CRC.

Kaas JH, Krubitzer LA (1991) Neuroanatomy of visual pathways and their retinotopic organization (Dreher B, Robinson SR, eds), pp 302-359. London: Macmillan.

Koyama M, Hasegawa I, Osada T, Adachi Y, Nakahara K, Miyashita Y (2004) Functional magnetic resonance imaging of macaque monkeys performing visually guided saccade tasks: comparison of cortical eye fields with humans. Neuron 41:795-807.

Larsen RJ, Marx ML (1986) An introduction to mathematical statistics and its applications, Ed 2. Englewood Cliffs, NJ: Prentice-Hall.

Lewis JW, Van Essen DC (2000) Mapping of architectonic subdivisions in the macaque monkey, with emphasis on parieto-occipital cortex. J Comp Neurol 428:79-111.

Lyon DC, Xu X, Casagrande VA, Stefansic JD, Shima D, Kaas JH (2002) Optical imaging reveals retinotopic organization of dorsal V3 in New World owl monkeys. Proc Natl Acad Sci USA 99:15735-15742.

Mazziotta JC, Toga AW, Evans AC, Fox PT, Lancaster JL (1995) Digital brain atlases. Trends Neurosci 18:210-211.

McKeefry DJ, Zeki S (1997) The position and topography of the human colour centre as revealed by functional magnetic resonance imaging. Brain 120:2229-2242.

Neuenschwander S, Gattass R, Sousa AP, Pinon MC (1994) Identification and visuotopic organization of areas PO and POd in Cebus monkey. J Comp Neurol 340:65-86.

Orban GA, Claeys K, Nelissen K, Smans R, Sunaert S, Todd JT, Wardak C, Durand JB, Vanduffel W (2006) Mapping the parietal cortex of human and non-human primates. Neuropsychologia, in press.

Pandya DN, Seltzer B (1982) Intrinsic connections and architectonics of posterior parietal cortex in the rhesus monkey. J Comp Neurol 204:196-210.

Pitzalis S, Sereno MI, Patria F, Committeri G, Galati G, Fattori P, Galletti C (2004a) A possible human homologue of the macaque V6. FENS Abstr 2: A052.18.

Pitzalis S, Sereno MI, Patria F, Committeri G, Galati G, Fattori P, Galletti C (2004b) A new retinotopic area in the human parietal occipital sulcus. NeuroImage 283:S38.

Pitzalis S, Galletti C, Patria F, Committeri G, Galati G, Fattori P, Sereno MI (2005) Functional properties of human visual area V6. NeuroImage 120:S23.

Portin K, Hari R (1999) Human parieto-occipital visual cortex: lack of retinotopy and foveal magnification. Proc R Soc Lond B Biol Sci 266:981-985.

Rosa MG, Tweedale R (2001) The dorsomedial visual areas in New World and Old World monkeys: homology and function. Eur J Neurosci 13:421-427.

Rosa MG, Tweedale R (2005) Brain maps, great and small: lessons from 
comparative studies of primate visual cortical organization. Philos Trans R Soc Lond B Biol Sci 360:665-691.

Rosa MGP, Schmid LM (1995) Visual areas in the dorsal and medial extrastriate cortices of the Marmoset. J Comp Neurol 359:272-299.

Rosa MGP, Tweedale R (2004) Maps of the visual field in the cerebral cortex of primates: functional organization and significance. In: The primate visual system (Kaas JH, Collins CE, eds), pp 261-288. Boca Raton, FL: CRC.

Sereno MI (1998) Brain mapping in animals and humans. Curr Opin Neurobiol 8:188-194.

Sereno MI, Allman JM (1991) Cortical visual areas in mammals. In: The neural basis of visual function (Leventhal AG, ed), pp 160-172. London: Macmillan.

Sereno MI, Tootell RBH (2005) From monkeys to humans: what do we now know about brain homologies? Curr Opin Neurobiol 15:135-144.

Sereno MI, McDonald CT, Allman JM (1994) Analysis of retinotopic maps in extrastriate cortex. Cereb Cortex 4:601-620.

Sereno MI, Dale AM, Reppas JB, Kwong KK, Belliveau JW, Brady TJ, Rosen BR, Tootell RB (1995) Borders of multiple visual areas in humans revealed by functional magnetic resonance imaging. Science 268:889-893.

Sereno MI, Pitzalis S, Martínez A (2001) Mapping of contralateral space in retinotopic coordinates by a parietal cortical area in humans. Science 294:1350-1354.

Sereno MI, Huang RS, Saygin A, Filimon F, Hagler D (2004) Retinotopy of human cortex using phase-encoded video. Paper presented at the 34th Annual Meeting of Society for Neuroscience, San Diego, October.

Simon O, Mangin JF, Cohen L, Le Bihan D, Dehaene S (2002) Topographical layout of hand, eye, calculation, and language-related areas in the human parietal lobe. Neuron 33:475-487.
Talairach J, Tournoux P (1988) Co-planar stereotaxic atlas of the human brain. New York: Thieme.

Tootell RB, Hadjikhani N (2001) Where is "dorsal V4" in human visual cortex? Retinotopic, topographic and functional evidence. Cereb Cortex 11:298-311.

Tootell RB, Reppas JB, Kwong KK, Malach R, Born RT, Brady TJ, Rosen BR, Belliveau JW (1995) Functional analysis of human MT and related visual cortical areas using magnetic resonance imaging. J Neurosci 15:3215-3230.

Tootell RB, Mendola JD, Hadjikhani NK, Ledden PJ, Liu AK, Reppas JB, Sereno MI, Dale AM (1997) Functional analysis of V3A and related areas in human visual cortex. J Neurosci 17:7076-7078.

Tootell RB, Hadjikhani N, Hall EK, Marrett S, Vanduffel W, Vaughan JT, Dale AM (1998) The retinotopy of visual spatial attention. Neuron 21:1409-1422.

Van Essen DC, Zeki SM (1978) The topographic organization of rhesus monkey prestriate cortex. J Physiol (Lond) 277:193-226.

Van Essen DC, Drury HA, Dickson J, Harwell J, Hanlon D, Anderson CH (2001) An integrated software suite for surface-based analyses of cerebral cortex. J Am Med Inform Assoc 8:443-459.

Watson JD, Myers R, Frackowiak RS, Hajnal JV, Woods RP, Mazziotta JC, Shipp S, Zeki S (1993) Area V5 of the human brain: evidence from a combined study using positron emission tomography and magnetic resonance imaging. Cereb Cortex 3:79-94.

Zarahn E, Aguirre GK, and D’Esposito M (1997) Empricial analyses of BOLD fMRI statistics. I. Spatially unsmoothed data collected under nullhypothesis conditions. NeuroImage 5:179-197.

Zeki SM (1978) The third visual complex of rhesus monkey prestriate cortex. J Physiol (Lond) 277:245-272. 